\title{
Optimization Research And Application Of Enterprise Website Based On Web Service
}

\author{
Hongyan Chen ${ }^{1}$, Junwei Wan ${ }^{1}$ and Zhao Jing ${ }^{2}$ \\ ${ }^{1}$ Beijing Institute of Tracking and Telecommunicaitons Technology,Beijing 100094, China \\ ${ }^{2}$ Nanjing University of Science and Technology School of Computer Science and Engineering, \\ Nanjing 210094, China \\ child_smile@163.com
}

\begin{abstract}
In order to solve the problem of the enterprise website access efficiency gradually declined, and resuting in poor quality of integrated service, This paper introduces the systematic and operability website performance optimization in detail, and then discusses the methods and techniques of the website performance optimization from server,application services,code,database and so on.It is proved that the website optimization methods is excellent,and gives the comparative analysis of website access performance before and after optimization.
\end{abstract}

Keywords: Web optimization, performance, database, fragmentation.

\section{Introduction}

In the internet + epoch, consumer uppermost is accepted by most enterprises. Website visiting speed and user capture experience value all are vital problems in any period of enterprise web site operation, It is the most basic key element in the friendly experience of website visiting; Otherwise any marketing popularization and service mode that the later stage does all might be made a futile effort.Because general client's patience is not high,The alternative costs that add client in the network are very low, and page access more than 6 seconds customers will leave, This is not a high flow of some of the enterprise website is even worse.

Website visiting experience value receives the user to use all factor affecting of product deeply.For example, Access to the application from the user to the content of the received feedback, Usually through DNS query, network transmission and access forwarding, WEB services, application services, middleware, database and other application components of information processing, the performance of these components will directly affect the realtime, accuracy and stability of business interaction. Therefore, face when enterprise web site under the situation of user experience value, to so many intermediate module link, the effective web site performance prioritization scheme of the system that how to propose will become an important research project.

This paper is from practical experience, To the website of long-term operation under the situation that access speed descends gradually, From server, application service, program and database etc. the Several Factors that access speed has the greatest impact is started with respectively.In line with preferential checking simple hypothesis, from simple to complicated, from the front end to the rear end, from outside to inside, the principle of step-by-step optimization.Provide a cover system, reality can operate, effectively improve the optimization web site performance scheme of user experience value,and the results of the optimized website access test are compared and analyzed.

\section{Performance optimization scheme design}

\subsection{Server optimization}

Usually the website is after operation a period of time.Continuous increase along with business,Constantly reaching the standard grade of new system applies,the Website server performance demands also progressively promotes, and server operation in the course of time runs 
into the performance bottleneck thing that also is inevitable, so we do the server optimization that web site performance optimization at first is exactly consideration.

Do not replace the new server, do not upgrade the hardware configuration, and does not increase the network bandwidth allocation, to solve the bottleneck of server performance situation, in order to protect the business is not affected, upgrade the server performance from two aspects:

1) Clear system log file regularly.The garbage system journal file can take the too many space of $C$ dish, therefore will regularly delete, and makes BAT or cmd running paper and clears up automatically, and the file script like Figure1.

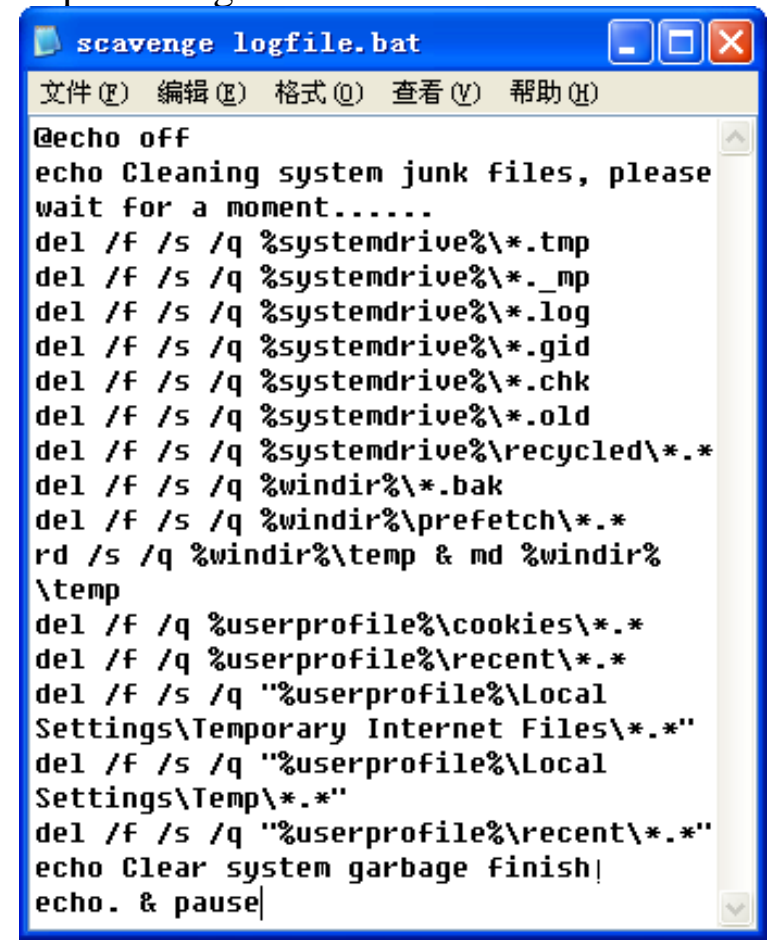

Fig. 1 Clear system log file script sketch map

2) disk fragments arrangement.Carry out defragmentation, fragment too much can make system seek back and forth when reading file, causes that systematic function descends, and needs periodic cleaning.

3) cleaning backup file.Can be with some the backup file deletions under the path"C: :Windows $\mid$ winsxs $\backslash$ Backup”, these files often take a large amount of disk spaces.

4) Clean Up Database error log file.The SQL2005 database,1433 ports owing to opened to the outside world,the hacker can attempt cracking the SA password, can cause producing a large amount of error log ERRORLOG files.and the error log path is "C:\Program Files\Microsoft SQL Server\MSSQL.1\MSSQL\LOG ”.

\subsection{Application service optimization}

Practice shows that the stand-by period major part of user capture webpage is to occur in the browser front-end, The practice shows that most of the waiting time for users to visit the web page is in the front end of the browser, especially when the page is loaded and a variety of elements is loaded (pictures, CSS scripts, JAVASCRIPT code, FLASH, etc.). Therefore, invest a lot of time and experience to improve the program, it is better to optimize the WEB front-end more effective.

1) Forbids unnecessary WEB service extension.IIS supports multiple service extension, principle be use what the expansion just launch what the expansion, some service extension not only takies the IIS resource influences performance, in addition exist security breaches, key be WEB in service just use at all less than.Forbid method: open the IIS manager, click "WEB service extension", choose corresponding expansion back to click "forbidding "and get final product.

2) Delete useless IIS extension mapping. IIS default support for.Asp and other extensions of the mapping, some useless extensions will increase the burden on the server and there is a certain security risk. Remove the mapping approach: open the IIS manager, "the default WEB site"in the 
"properties"tab, open the "configuration" window, select the extension to delete the map after the click “delete”.

3) Cancel access record. IIS default open WEB access records are numerous, and occupy a large amount of disk space, seriously affect the performance of WEB server. Cancel access records of the operation: open the IIS manager, to find the specific WEB site, open the"properties"tab, in the "main directory"to cancel the "record access"check can be.

4) Limit access to traffic. Open IIS manager, targeted to the specific WEB site, open the “properties”window, select "properties”tab, select “enable bandwidth limit”, in "the largest network use"settings, set specific values of bandwidth.

5) Let WEB load free flex. The operation step is: in the "Internet information services"in the management of the application pool "properties"dialog box, open the "performance"tab, in the "maximum number of working process"input box input process.

6) Clear cache file. Manually clear the cache file "C:IWinnt $\backslash I I S$ Temporary Compressed Files", when the IIS website launched gzip function, page access will produce a large number of files in the $\mathrm{C}$ dish, will lead to full the $\mathrm{C}$ dish.

7) Build an independent application pool and set it to exclusive access.

\subsection{Program optimization}

Through the optimization of the program code, in the database connection to add some skills, just can let program carry out quickly, can make to use to reach optimum performance.

\subsubsection{Site scripting optimization}

1) The data of often using are buffered on the WEB server. Such as the reference list, menu items, the Extended Markup Language (XML) string, the combo box list, DHTML fragmentation and site configuration variables (Internet protocol, IP address, including the name of the data source DSN, and WEB path), etc..

2) The data of often using are buffered among Session object or the Application. Session objects and Application ASP provide a convenient cache container for commonly used data between HTTP calls.

3) Defined variable will define private variable, and private variable is in the speed of service and faster than global variable speed above the space hold.Use the function return character, try not to use variable, use private variable as far as possible if must use variable.

4) In the first place ASP program <\% option explicit\% > VBScript statement variables, and avoid server parses affect server request response speed.

5)Use case to select the conditions, avoid using the if statement.

6) Avoid carrying out object definition in using the global.asa file.

7) Use Response.Write to generate HTML code, to maintain the continuity of the ASP code to improve performance, to avoid the spread of ASP code and HTML language commands.

8) Use $<$ OBJECT $>$ tags instead of Server.CreateObject. $<$ OBJECT $>$ is created when the object is referenced for the first time, and the latter will cause the object to be created immediately to improve efficiency.

9) Open the "Explicit Option" explicitly defining variable options, avoiding the creation of variables across the entire namespace search.

10) Using Server.MapPath, using MapPath text clear path.

11) Page execution before the use of Response.Is Client Connected to avoid the page for a long time to complete the load.

12) Used in the URL directory after the slash (/).

13) Use Server.Transfer at any time, rather than using Response.Redirect to optimize the steering link.

\subsubsection{Database access optimization}

1) When the object is used, first use the Close method to release the system resources occupied by the object, and then set the object value for the "nothing" to release the object occupied memory.

2) Through the use of stored procedures to access the database, with the "Data Objects ActiveX (ADO)”, will win a lot of program performance. 
3) When only take the front of $\mathrm{N}$ records, do not use "select *", and then ADO paging, to "select top N" field list will be much more efficient.

4) When the number of statistical records do not use "select * from..." such a statement, switch to "count from (1) select..”.

5) Try to avoid the use of "select* from table"such a SQL statement, will cause the program to read too many useless data from the database, you should use the "select field1, field2 from table" in this form, only to read the necessary data from the database.

6) Do not use nested queries, should be used "SQL =select a.id ,b.name from a left join b on b.id=a.id” such a table operation, and use the specific field name instead of *.

7) Data query to avoid using the "NOT IN (...)"this statement, the efficiency will improve a lot.

8) Remove unnecessary queries using the statement” SELECT *”, “Order by”, "group by”, etc..

9)Remove unnecessary program lock;

10) Modify unreasonable expand the scope of inquiry statements, use the $<,<>$, $>$,"not exists" instead of" not in" etc..

11) No sub query.

12) Execute the statement to avoid the four operation of the column.

13) Insert, update, delete, and other changes to the statement on the end of the program before the implementation, to reduce the possibility of deadlock.

\subsection{Database optimization}

For operating website, as time passes, the frequent use of application system,Database is in daily use, Continuous insert, delete, update operation,Causing showing fragment occurring with index is the thing that can hardly be avoided, and will become the main factor that influences website reaction speed and performance. The fragment of database can cause systematic function to weaken and waste a large amount of table spaces, optimizes database, promotes the effective means of user experience value.

Many data blocks under the high-water line (HWL) all are free of data, but will scan the data block of high-water line full table scan the time, that is to say that oracle will do many idle works!Therefore oracle provides shrink space defragmentation function.

1) Backup sheet $A$ is to backup sheet $B$, and backup is the prerequisite of defrag:

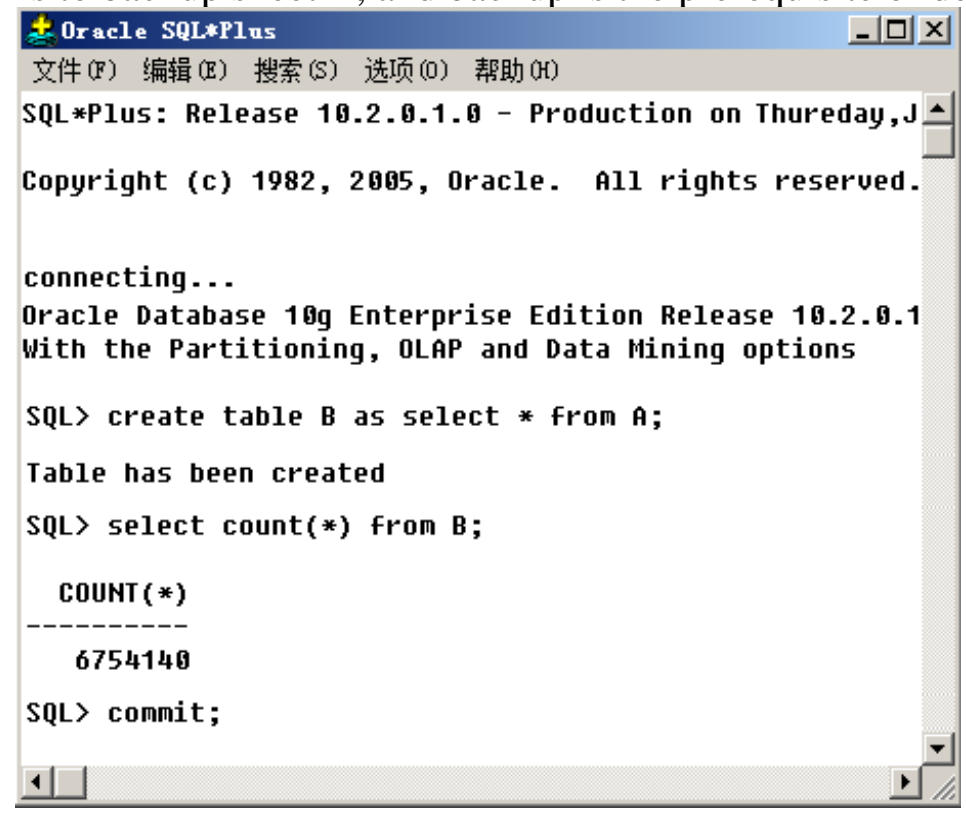

Fig.2 Oracle Database table backup operation command

2) Analysis table A.

Return the results as shown in Figure3, and how many spaces estimating table also has to use under high-water line, and this value should be low more good more, and the table utilization rate is more near high-water line, and the idle work that full table scan is done is also just few more. 


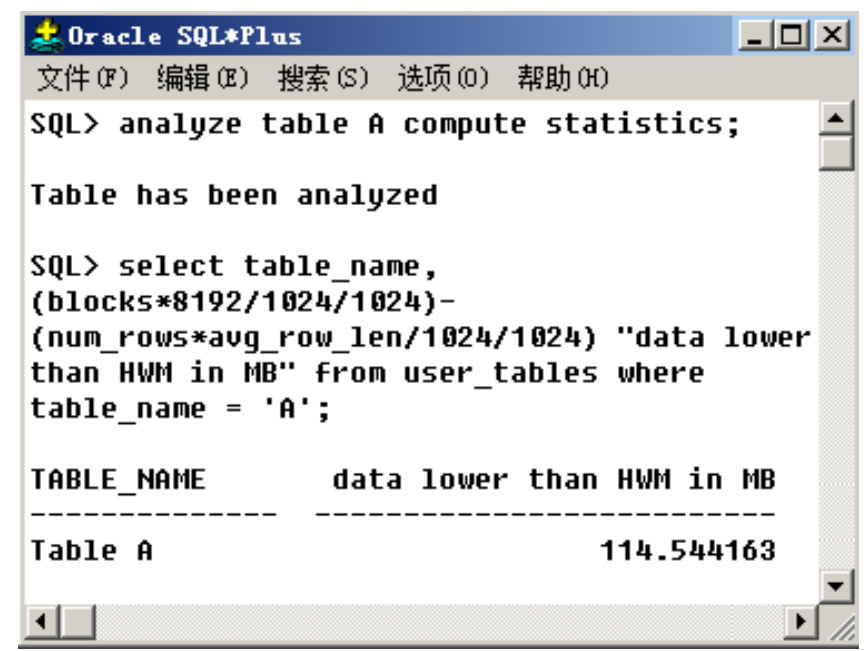

3) Database fragment.

Fig.3 Oracle Database table analysis operation command

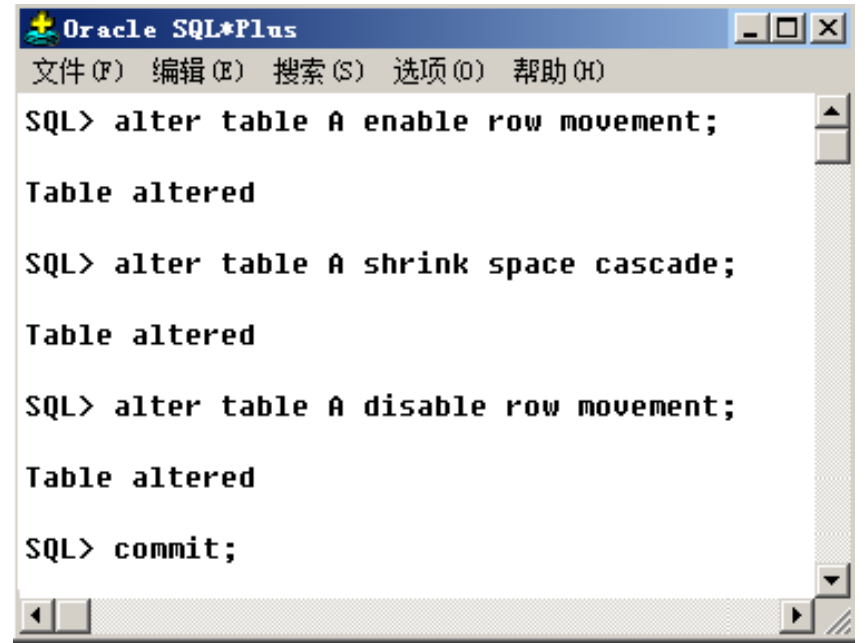

Fig.4 Oracle Database table fragment operation command

4) If the table has been created before, then the relevant view needs to be re compiled, and then restart the database, and then all of the finished.

As one of the most important factors that affect the performance of the database, the database fragmentation should be paid enough attention to by the network administrator.

\subsection{Other optimization}

1) Mobile device priority, a single data object is less than $25 \mathrm{~K}$. At present, the internet+epoch, mobile Internet is becoming more and more popular, so the site link objects smaller load faster, the mobile user experience more excellent.

2)Do not use zoom. Try to make the right size of the picture, avoid using the ImageMagic command (convert) to stretch or compress the picture, after all, the user to see too much stretch the picture is very ugly.

3) Optimized picture. Compared to GIF, JPG, PNG format pictures, as far as possible the use of PNG format pictures, he has more features and smaller size.

4) Control third party scripts. After the content of the key page to load the third party script, the more ideal is placed on the page after the onLoad.

\section{Performance test and analysis}

After the optimization of the Website have been done, and then restart the Website server can clearly see the optimization effect. Through the WEB performance testing tool to test, test results shown in Figure 5 and Figure 6, the acquisition of the HTML document, the optimized time spent less than $20 \%$ of the total response time before optimization, page loading time is $365 \mathrm{~ms}$, HTML generated 
page time is greatly shortened, the background time reduced by 50\%. (test using third party WEB tools, all test results are carried out under the third party browser local 0 cache).

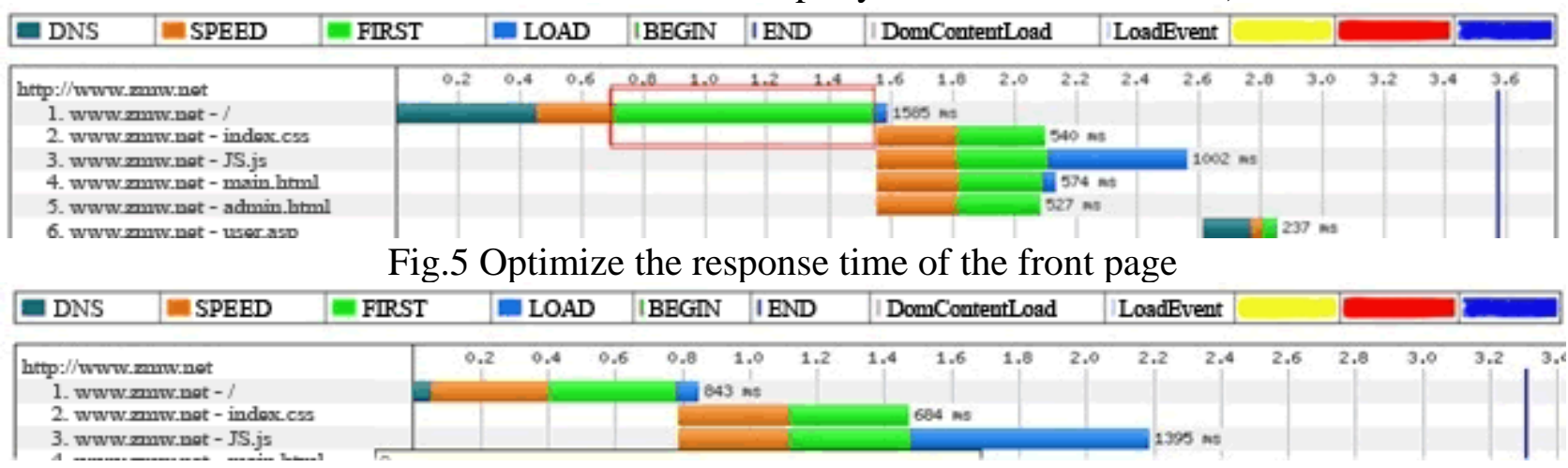

Fig.6 The optimized response length was significantly shortened

On the home page of the site for a period of time monitoring, loading speed test shows the results shown in Figure 7, the loading time is significantly shortened, the overall performance of the website to achieve a greater.

History Graph -Home Page Load Time (2016/10/19 00:00 - 2016/10/25 16:00) \.



Fig.7 Home monitor display

\section{Summary}

Performance optimization is a complex topic, which involves a lot of things, to optimize the whole process needs to take into account a lot of factors. This paper summarized the optimization methods for a large site, is a very common and very effective solutions, but the implementation of the different business, will achieve their way of realization, here refers to the combination of different schemes and corresponding technical methods.

The performance factor is a website system development one of the important driving force, careful thinking and are difficult to compatible so many unknown scenes, well in scalability and compatibility on the work site to avoid cold pain, heat pain more website.

In addition, there is one of the most critical issues, before doing any optimization and operation, please do a good job of website and database backup.

\section{References}

[1] Berkeley College. Web Traces and Logs. http://www.web-caching.com/traces-logs.html, 2012-07-18

[2] Liu B, Shi H, Shi L, et al. A Web Predictive Model Based on Dependency Graph. Journal of Software, 2011, 6(9):1829-1836

[3] Ali W, Shamsuddin S M, Ismail A S. A survey of Web caching and prefetching. Soft Comput, 2011,3(1):18-44 
[4] Qian F, Quah K S, Huang J, et al. Web caching on smartphones: Ideal vs. reality. In: Proceedings of the 10th international conference on Mobile systems, applications, and services. Santa Cruz, 2012, $127-140$

[5] Andresen D,Tso Yang,Ibarra O H SWEB:Towards a scalehic World wide Web selection muhicomputers[C].Proceeding at the 10th International Parallel Processing Symposium

[6] K.McCloghrie,M.Rose.Management Information Base for Network Management of TCP-/I-based internets [RFC 1156]

[7] Qiang Yang, Henry Hanning Zhang. Integrating Web Prefetching and Caching Using Prediction Models. World Wide Web, 2011, 4(4):299-321

[8] Montoto P, Pan A, Raposo J, et al. Automated browsing in AJAX websites. Data and Knowledge Engineering, 2011, 70(3):269-283

[9] Chen J B. Efficient Content Placement on Multimedia CDN Using Fuzzy Decision Algorithm. APPLIED MATHEMATICS, 2012, 6(2):471-477

[10] Singh D, Kumar S, Kapoor S. An Explore View of Web Caching Techniques. International Journal of Advances in Engineering Sciences, 2011, 1(3):38-43

[11] Simtec Limited. HttpWatch Professional. http://www.httpwatch. com/download/, 2012-05-14

[12] Goel N, Martery S, Kanauziya S. Web Technologies. International Journal of Computer Applications, 2010, 1(24):11-21 